\title{
POSITIVE BOUND STATES HAVING PRESCRIBED SYMMETRY FOR A CLASS OF NONLINEAR ELLIPTIC EQUATIONS IN $\mathbf{R}^{N}$
}

\author{
Florin CATRINA, Zhi-Qiang WANG \\ Department of Mathematics and Statistics, Utah State University, Logan, UT 84322, USA
}

Received in 20 December 1999

ABSTRACT. - We consider positive solutions of

$$
-\operatorname{div}\left(|x|^{-2 a} \nabla u\right)=|x|^{-b p} u^{p-1}, \quad u \geqslant 0 \text { in } \mathbf{R}^{N},
$$

where for $N \geqslant 2: a<(N-2) / 2, a<b<a+1$, and $p=2 N /(N-2(1+a-b))$. Ground state solutions are the extremal functions of the Caffarelli-Kohn-Nirenberg inequalities [6]. In [10] the authors have observed symmetry breaking phenomena for ground state solutions in a subregion of the parameters. In this paper, we continue our study on the structure of bound state solutions and construct bound state solutions having prescribed symmetry.

(C) 2001 L'Association Publications de l'Institut Henri Poincaré. Published by Elsevier B.V. All rights reserved

Keywords: Positive solutions; Degenerate elliptic equations; Prescribed symmetry; Local minimizations

RÉSUMÉ. - On considère les solutions positive à

$$
-\operatorname{div}\left(|x|^{-2 a} \nabla u\right)=|x|^{-b p} u^{p-1}, \quad u \geqslant 0 \text { dans } \mathbf{R}^{N},
$$

où, pour $N \geqslant 2$ : $a<(N-2) / 2, a<b<a+1$, et $p=2 N /(N-2(1+a-b))$. Les solutions à état fondamental sont les fonctions extrémales pour les inégalités des Caffarelli-KohnNirenberg [6]. Dans [10] les auteurs ont observé une brisure de symétrie pour les solutions d'état fondamental dans une région des paramètres. L'objet de cet article est l'étude de la structure des solutions à état borné et de construire des solutions à état borné avec une symétrie prescrite. (C) 2001 L'Association Publications de l'Institut Henri Poincaré. Published by Elsevier B.V. All rights reserved

\section{Introduction}

This paper is concerned with the following class of problems,

$$
-\operatorname{div}\left(|x|^{-2 a} \nabla u\right)=|x|^{-b p} u^{p-1}, \quad u \geqslant 0 \text { in } \mathbf{R}^{N},
$$


where

$$
N \geqslant 2, \quad a<\frac{N-2}{2}, \quad a<b<a+1 \quad \text { and } \quad p=\frac{2 N}{N-2(1+a-b)} .
$$

The motivations for our study are twofold. First, Eq. (1) is a prototype of more general degenerate elliptic equations:

$$
-\operatorname{div}(\alpha(x) \nabla u)=f(x, u), \quad x \in \mathbf{R}^{N},
$$

which are models for some physical phenomena related to equilibrium of anisotropic continuous media which possibly are somewhere perfect insulators and somewhere are perfect conductors (e.g., [13]). Though there is a quite extensive literature about degenerate elliptic operators, only few recent results about nonlinear variational problems associated to degenerate elliptic equations like (1) are known (e.g., [2,7] and references therein). On the other hand, Eq. (1) is related to the following inequalities due to Caffarelli, Kohn, and Nirenberg [6]. With $a, b$, and $p$ in the range above, there is a positive constant $C_{a, b}$ such that for all $u \in \mathcal{D}_{a}^{1,2}\left(\mathbf{R}^{N}\right)$

$$
\int_{\mathbf{R}^{N}}|x|^{-2 a}|\nabla u|^{2} \mathrm{~d} x \geqslant C_{a, b}\left(\int_{\mathbf{R}^{N}}|x|^{-b p}|u|^{p} \mathrm{~d} x\right)^{2 / p} .
$$

By $\mathcal{D}_{a}^{1,2}\left(\mathbf{R}^{N}\right)$ we mean the completion of $C_{0}^{\infty}\left(\mathbf{R}^{N}\right)$ with respect to the inner product

$$
(u, v)=\int_{\mathbf{R}^{N}}|x|^{-2 a} \nabla u \cdot \nabla v \mathrm{~d} x .
$$

Let

$$
E_{a, b}(u)=\frac{\int_{\mathbf{R}^{N}}|x|^{-2 a}|\nabla u|^{2} \mathrm{~d} x}{\left(\int_{\mathbf{R}^{N}}|x|^{-b p}|u|^{p} \mathrm{~d} x\right)^{2 / p}}
$$

and

$$
S(a, b)=\inf _{u \in \mathcal{D}_{a}^{1,2}\left(\mathbf{R}^{N}\right) \backslash\{0\}} E_{a, b}(u),
$$

the best embedding constants for the Caffarelli-Kohn-Nirenberg inequalities. The extremal functions which achieve $S(a, b)$ are ground state solutions of (1). There have been extensive studies on the ground state solutions of (1). The existence of extremal functions for $S(a, b)$ has been given for $a \geqslant 0$ (which occurs only for $N \geqslant 3$ ), and $a \leqslant b<a+1$, as follows. The case $a=0$ and $b=0$, was settled by Aubin [1], and Talenti [26]. The segment $a=0,0<b<1$ was dealt with by Lieb in [20], and the region $0<a<(N-2) / 2, a \leqslant b \leqslant a+1$ in [11,28,22]. For $a \geqslant 0$, the conclusion is that there are no bound state solutions other than the ground state solutions and that the ground state solutions are unique up to a dilation

$$
u(x) \rightarrow \tau^{\frac{N-2-2 a}{2}} u(\tau x), \quad \tau>0,
$$


and are radial (in the case $a=b=0$, solutions are radial with respect to some point) and explicitly given. For the case $a<0$, little has been known until the recent work in [7, $10,16,30]$. We have studied the ground state solutions in [10] and have established the following.

(i) For $a=b<0, S(a, a)$ is not achieved, i.e. there is no ground state solution.

(ii) For $a<0$ and $a<b<a+1, S(a, b)$ is always achieved.

(iii) There is a function $h(a)$ defined for $a \leqslant 0$ satisfying $h(0)=0, a<h(a)<a+1$ for $a<0$, and $a+1-h(a) \rightarrow 0$ as $a \rightarrow-\infty$, such that in the region $a<0$, $a<b<h(a)$, the ground state solution is nonradial.

(iv) All bound state solutions of (1) satisfy a modified inversion symmetry.

Since radial solutions exist for all $a \leqslant b<a+1$, our result shows that for $a<0$ there is a region where bound state solutions of (1) are not unique (even up to dilations). Thus it is a fundamental issue to investigate the solutions structure for problem (1). The goal of this paper is to give existence of bound state solutions having prescribed symmetry $G$, where $G$ is a subgroup of the orthogonal group $\mathbf{O}(N)$. Note that (1) is radially invariant, in the sense that if $u$ is a solution, so is $g u$ for all $g \in \mathbf{O}(N)$, where

$$
g u(x)=u\left(g^{-1} x\right) .
$$

A consequence of our main result is the following

THEOREM 1.1. - For $b-a \in(0,1)$ fixed, for any $k \in \mathbf{N}$ there is $a_{k}<0$ such that for $a \leqslant a_{k}$ problem (1) has $k$ essentially distinct solutions.

By essentially distinct solutions, we mean functions which can not be obtained from one another by some transformation which leaves the problem invariant.

Our results also partially answer the question whether one can classify all solutions of (1) according to their symmetry. Here, for $u \in \mathcal{D}_{a}^{1,2}\left(\mathbf{R}^{N}\right)$, the symmetry group of $u$ is defined to be

$$
\Sigma_{u}=\{g \in \mathbf{O}(N): g u=u \text { a.e. }\} .
$$

To state our main results, let us make the following

Definition 1.2. - Let $G \subset \mathbf{O}(N)$ be a closed subgroup, and fix an action of $G$ on $\mathbf{S}^{N-1}$. We say that the action of $G$ has a locally minimal orbit set $\Omega \subset \mathbf{S}^{N-1}$, if there exist $k \in \mathbf{N}$ and $\delta>0$, such that

(a) $\Omega$ is $G$-invariant,

(b) $\# G y=k$ for any $y \in \Omega$,

(c) $\# G y>k$ for any $y \in \mathbf{S}^{N-1}$ with $0<\operatorname{dist}(y, \Omega)<\delta$.

Here $G y$ is the orbit of $y$ under the action of $G$, and \#Gy is the cardinal number of $G y$.

DEFINITION 1.3. - We say that the action of $G$ is maximal with respect to a locally minimal orbit set $\Omega$ if for any closed subgroup $H$, with $G \leqslant H \leqslant \mathbf{O}(N), H \neq G$, we

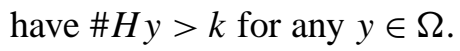

Our main theorem is 
THEOREM 1.4. - Assume $G \subset \mathbf{O}(N)$ is a closed subgroup having a locally minimal orbit set $\Omega \subset \mathbf{S}^{N-1}$ (corresponding to an integer $k$ ). Let $b-a \in(0,1)$ fixed, then for $-a$ sufficiently large, (1) has a solution $u_{a}$ satisfying

(i) $u_{a}$ is $G$-invariant,

(ii) $u_{a}$ satisfies the modified inversion symmetry $u_{a}\left(x /|x|^{2}\right)=|x|^{N-2-2 a} u_{a}(x)$,

(iii) $E_{a, b}\left(u_{a}\right)=((N-2-2 a) / 2)^{2(b-a)}\left(k^{(p-2) / p} S_{p}\left(\mathbf{R}^{N}\right)+\mathrm{o}(1)\right)$, as $a \rightarrow-\infty$,

(iv) $u_{a}$ is a k-bump solution, concentrating near $\Omega$ as a $\rightarrow-\infty$,

(v) if in addition, $G$ is maximal with respect to $\Omega$, then $\Sigma_{u}=G$.

In (iii), we take $S_{p}\left(\mathbf{R}^{N}\right)$ to be the best constant in the embedding of $H^{1}\left(\mathbf{R}^{N}\right)$ into $L^{p}\left(\mathbf{R}^{N}\right)$, i.e.

$$
S_{p}\left(\mathbf{R}^{N}\right)=\inf _{u \in H^{1}\left(\mathbf{R}^{N}\right) \backslash\{0\}} \frac{\int_{\mathbf{R}^{N}}|\nabla u|^{2}+u^{2} \mathrm{~d} x}{\left(\int_{\mathbf{R}^{N}}|u|^{p} \mathrm{~d} x\right)^{2 / p}} .
$$

By $k$-bump solutions concentrating near $\Omega$ as $a \rightarrow-\infty$, we mean the following: there is $\left\{y_{1}, \ldots, y_{k}\right\} \subset \Omega$, a $G$-orbit, such that for any $r>0$,

$$
\lim _{a \rightarrow-\infty} \int_{\mathbf{R}^{N} \backslash \bigcup_{i=1}^{k} B_{r}\left(y_{i}\right)}|x|^{-2 a}\left|\nabla u_{a}\right|^{2} \mathrm{~d} x=\lim _{a \rightarrow-\infty} \int_{\mathbf{R}^{N} \backslash \bigcup_{i=1}^{k} B_{r}\left(y_{i}\right)}|x|^{-b p} u_{a}^{p} \mathrm{~d} x=0 .
$$

Remark 1.5. - In fact, we can give a more precise asymptotic behavior of solutions $u_{a}$. We look first at the equation

$$
-\Delta u+u=u^{p-1}, \quad u>0 \text { in } \mathbf{R}^{N} .
$$

Let $\left(z_{1}, \ldots, z_{N}\right)$ be coordinates in $\mathbf{R}^{N}$ and $u(z)$ the unique solution (centered at 0 ) of (4). Then note that for $b-a \in(0,1)$ fixed, as $a \rightarrow-\infty$, the function

$$
v_{a}(z):=\left(\frac{N-2-2 a}{2}\right)^{\frac{N-2(1+a-b)}{2(1+a-b)}} u\left(\frac{N-2-2 a}{2} z\right)
$$

concentrates at the origin. Now, for $\left\{y_{1}, \ldots, y_{k}\right\} \subset \Omega$ a $G$-orbit, consider the projections

$$
\pi_{i}(x)=\frac{x-\left(x \cdot y_{i}\right) y_{i}}{|x|}, \quad i=1, \ldots, k .
$$

For $r>0$ small and $\left|x-y_{i}\right|<r$, we define

$$
u_{a, i}(x)=|x|^{-\frac{N-2-2 a}{2}} v_{a}\left(\pi_{i}(x),-\ln |x|\right) .
$$

Then we can show that the distance between $u_{a}$ and $\sum_{i=1}^{k} u_{a, i}$ tends to 0 in $\mathcal{D}_{a}^{1,2}\left(\mathbf{R}^{N}\right)$, as $a \rightarrow-\infty$.

Remark 1.6. - Since the problem is invariant under dilations (3), we can in fact obtain solutions of $k$-bump type with bumps concentrating near $r \Omega \subset \mathbf{S}^{N-1}(r)$, for any $r>0$. 
Remark 1.7. - Depending on the action of $G$, we obtain two types of solutions: solutions which are absolute minimizers in the space of symmetric functions (in particular, ground state solutions when $k=1$ ), and solutions which are local minimizers in the space of symmetric functions. We obtain both, by a rather general local minimization method used in [8] and [27]. We should mention that global minimization methods in symmetric subspaces have been used by Coffman in [12] for $N=2$, and Li in [19] for $N \geqslant 4$ to study a problem in expanding annular domains. We developed the local minimization method in [8] to solve the case $N=3$ and to construct solutions with prescribed symmetry.

Our method involves two ingredients: a transformation that converts Eq. (1) to another one defined on a cylinder $\mathcal{C}=\mathbf{S}^{N-1} \times \mathbf{R}$ in $\mathbf{R}^{N+1}$ and its dilations $\mathcal{C}_{\lambda}$, (this transformation was used in our previous work [10] for studying ground state solutions); and a local minimization scheme which enables us to obtain bound states as local minimizers, (this approach has been used in our previous work [8,27], to construct positive solutions with prescribed symmetry for other types of nonlinear elliptic problems). In Section 2 we give the equivalent problem, setting up the stage for studying new Eq. (8). Section 3 is devoted to some asymptotic analysis by using the concentration-compactness principle. Then in Section 4 we give the proof of the results for Eq. (8) which in turn will give our main results for Eq. (1). Some of the results in this paper as well as those in [10] have been announced in [9].

\section{Preliminaries}

\subsection{Equivalent problems}

Denote $\mathcal{C}=\mathbf{S}^{N-1} \times \mathbf{R}$ the cylinder in $\mathbf{R}^{N+1}$. Throughout, whenever the situation arises, we use the notations in Riemannian geometry,

$$
\begin{gathered}
\Delta v=g^{i j} v_{i j}-g^{i j} \Gamma_{i j}^{k} v_{k} \quad \text { the Laplace-Beltrami operator, } \\
\nabla v=g^{i j} v_{j} \partial_{i} \quad \text { the gradient, } \\
\operatorname{div}\left(X^{i} \partial_{i}\right)=\frac{1}{\sqrt{g}}\left(\sqrt{g} X^{i}\right),_{i}=X^{i},{ }_{i}+\Gamma_{i j}^{i} X^{j}
\end{gathered}
$$

and

$$
\mathrm{d} \mu \text { the volume element, }
$$

all corresponding to the metric $g_{i j}$ induced from $\mathbf{R}^{N+1}$.

In [10] we have shown that with suitable transformations, problem (1) is equivalent to another differential equation on $\mathcal{C}$. We take the same approach here and we shall mainly work with the equivalent problem on $\mathcal{C}$. Let us make this more precise. As it was shown in [10], by the transformation

$$
u(x)=|x|^{-\frac{N-2-2 a}{2}} v\left(\frac{x}{|x|},-\ln |x|\right),
$$


162 F. CATRINA, Z.-Q. WANG / Ann. Inst. Henri Poincaré, Anal. non linéaire 18 (2001) 157-178

problem (1) is equivalent to

$$
-\Delta v+\left(\frac{N-2-2 a}{2}\right)^{2} v=v^{p-1}, \quad v>0 \text { on } \mathcal{C} .
$$

In fact, the mapping (5) is a Hilbert space isomorphism from $\mathcal{D}_{a}^{1,2}\left(\mathbf{R}^{N}\right)$ to $H^{1}(\mathcal{C})$ (Proposition 2.2, [10]). I.e., if $u$ defined on $\mathbf{R}^{N}$ and $v$ defined on $\mathcal{C}$ are related through (5), then $u \in \mathcal{D}_{a}^{1,2}\left(\mathbf{R}^{N}\right)$ if and only if $v \in H^{1}(\mathcal{C})$, and in this case

$$
E_{a, b}(u)=F_{a, b}(v):=\frac{\int_{\mathcal{C}}|\nabla v|^{2}+\left(\frac{N-2-2 a}{2}\right)^{2} v^{2} \mathrm{~d} \mu}{\left(\int_{\mathcal{C}}|v|^{p} \mathrm{~d} \mu\right)^{2 / p}} .
$$

To study (6), we shall further transform it to another equivalent problem. Let us denote

$$
\lambda=\frac{N-2-2 a}{2},
$$

and consider the similarity transformation $h_{\lambda}: \mathbf{R}^{N+1} \rightarrow \mathbf{R}^{N+1}$ given by

$$
h_{\lambda}(x)=\lambda x .
$$

Denote $\mathcal{C}_{\lambda}=h_{\lambda}(\mathcal{C})$ and for $v \in H^{1}(\mathcal{C})$, we define $w \in H^{1}\left(\mathcal{C}_{\lambda}\right)$ by

$$
v(x)=\lambda^{2 /(p-2)} w(\lambda x) .
$$

Then

$$
F_{a, b}(v)=\lambda^{2(b-a)} \frac{\int_{\mathcal{C}_{\lambda}}|\nabla w|^{2}+w^{2} \mathrm{~d} \mu}{\left(\int_{\mathcal{C}_{\lambda}}|w|^{p} \mathrm{~d} \mu\right)^{2 / p}},
$$

and with the rescaling (7), problem (6) is equivalent to

$$
-\Delta w+w=w^{p-1}, \quad w>0 \text { in } \mathcal{C}_{\lambda} .
$$

In the following we shall find critical points of

$$
I_{\lambda}(v)=\int_{\mathcal{C}_{\lambda}}|\nabla v|^{2}+v^{2} \mathrm{~d} \mu
$$

for $v \in \mathcal{M}_{\lambda}$ in $H^{1}\left(\mathcal{C}_{\lambda}\right)$, where

$$
\mathcal{M}_{\lambda}=\left\{\left.v \in H^{1}\left(\mathcal{C}_{\lambda}\right)\left|\int_{\mathcal{C}_{\lambda}}\right| v\right|^{p} \mathrm{~d} \mu=1\right\} .
$$

By rescaling they are critical points of $F_{a, b}$ in $H^{1}(\mathcal{C})$ and by (5) we obtain critical points of $E_{a, b}$. 


\subsection{Group actions}

Let

$$
\psi: G \times \mathbf{S}^{N-1} \rightarrow \mathbf{S}^{N-1},
$$

the action of a closed subgroup $G$ of $\mathbf{O}(N)$. We consider

$$
\mathcal{C}=\left\{\left(x_{0}, \ldots, x_{N}\right) \in \mathbf{R}^{N+1}: x_{0}^{2}+\cdots+x_{N-1}^{2}=1\right\} .
$$

If we consider the unit sphere $\mathbf{S}^{N-1}=\left\{x \in \mathcal{C} \mid x_{N}=0\right\}$, Definitions 1.2 and 1.3 translate on $\mathcal{C}$ in a direct way. In the following, we shall impose the reflection symmetry $\mathbf{O}(1)$ about the hyperplane $x_{N}=0$, and we look for solutions invariant under the augmented group $\tilde{G}=G \times \mathbf{O}(1) \subset \mathbf{O}(N+1)$. This does not restrict the problem in any way since we showed in [10] that any positive solution of (6) has this reflection symmetry.

Next, we give examples of locally minimal orbit sets for a $G$ action on $\mathbf{S}^{N-1} \subset \mathcal{C}$.

Let $N=2$.

Example 2.1. - For $k \geqslant 2$, consider $G=\mathbf{Z}_{k}$ the group of rotations by multiples of $2 \pi / k$ acting in the plane $\left(x_{0}, x_{1}\right)$. Then

$$
\Omega=\left\{\left(x_{0}, x_{1}, 0\right): x_{0}^{2}+x_{1}^{2}=1\right\},
$$

is a locally minimal orbit set.

Example 2.2. - For $k \geqslant 3$, we consider $G=D_{k}$, the group that leaves invariant a regular polygon with $k$ vertices. When we take $\Omega_{1}$ to be the set of vertices, or $\Omega_{2}$ to correspond to the set of centers of the edges, both $\Omega_{1}$ and $\Omega_{2}$ are locally minimal orbit sets. In fact, $\Omega_{3}=\Omega_{1} \cup \Omega_{2}$ is also a locally minimal orbit set. In this case, the action of $G$ is maximal with respect to each $\Omega_{i}$.

Let $N=3$.

Example 2.3. - Consider $G$ to be the group which leaves invariant one of the regular polyhedra: a regular tetrahedron, octahedron, or icosahedron. Accordingly, we may take $\Omega_{0}$ to be the set of vertices, $\Omega_{1}$ to be the set of the centers of the edges, and $\Omega_{2}$ the set of the centers of the faces. In each of the three cases for tetrahedron, octahedron, or icosahedron, $\Omega_{i}$ for $i=0,1,2$ are locally minimal orbit sets, and $G$ is maximal with respect to $\Omega_{0}$. For the case of tetrahedron, $G$ is also maximal with respect to $\Omega_{2}$.

Example 2.4. - In this example, write $\mathbf{R}^{3}=\mathbf{R}^{2} \times \mathbf{R}$, and $G=\mathbf{Z}_{k} \times \mathbf{Z}_{2}$ or $G=$ $D_{k} \times \mathbf{Z}_{2}$. Then

$$
\Omega=\left\{\left(x_{0}, x_{1}, 0,0\right): x_{0}^{2}+x_{1}^{2}=1\right\},
$$

or $\Omega$ is the set of vertices of a regular polygon in the plane $\left(x_{0}, x_{1}\right)$, respectively. In both these cases, $\Omega$ is a locally minimal orbit set. In the case of $G=D_{k} \times \mathbf{Z}_{2}, G$ is maximal with respect to $\Omega$. 
Example 2.5. - One case is to consider finite subgroups of $\mathbf{O}(N)$ which act irreducible on $\mathbf{R}^{N}$.

Example 2.6. - On the other hand, many more examples can be obtained as follows. For $1 \leqslant l \leqslant N-1$ we make the decomposition $\mathbf{R}^{N}=\mathbf{R}^{l} \times \mathbf{R}^{N-l}$, and we look at groups $G=G_{l} \times G_{N-l}$, where $G_{l}$ acts irreducible on the $\mathbf{R}^{l}$ part, while the action of $G_{N-l}$ has no fixed points in $\mathbf{R}^{N-l}$, otherwise arbitrary. In particular, one can take $l$-dimensional tetrahedron and cube groups for $G_{l}$. Let $k:=\min \left\{\# G y: y \in \mathbf{S}^{l-1}\right\}$, and $\Omega_{l} \subset \mathbf{S}^{l-1}$ a locally minimal orbit set corresponding to this $k$. Then $\Omega:=\Omega_{l} \times\{0\}$, is a locally minimal orbit set for the action of $G$, with the same $k$.

\subsection{Local identifications}

We define a diffeomorphism between the ball of radius $r$ centered at the origin in $\mathbf{R}^{N}$ and a subset of $\mathcal{C}_{\lambda}$ as follows. We identify $\mathbf{R}^{N}$ with the tangent space to $\mathcal{C}_{\lambda}$ at $y$ and consider the projection in $\mathbf{R}^{N+1}$ in the direction of the normal to $\mathcal{C}_{\lambda}$ at $y$.

To be exact, let

$$
\mathcal{C}_{\lambda}=\left\{\left(x_{0}, \ldots, x_{N}\right) \in \mathbf{R}^{N+1}: x_{0}^{2}+\cdots+x_{N-1}^{2}=\lambda^{2}\right\} .
$$

Assume $y=\left(\lambda, 0, \ldots, 0, y_{N}\right) \in \mathcal{C}_{\lambda}$ and for $0<r<\lambda$, define a map from

$$
B_{\lambda, r}(y):=\left\{x \in \mathcal{C}_{\lambda}: x_{1}^{2}+\cdots+x_{N-1}^{2}+\left(x_{N}-y_{N}\right)^{2}<r^{2}\right\},
$$

onto $B_{r}(0) \subset \mathbf{R}^{N}$, by

$$
\phi_{\lambda, r, y}(x)=\left(x_{1}, \ldots, x_{N-1}, x_{N}-y_{N}\right) \in \mathbf{R}^{N} .
$$

For any $y \in \mathcal{C}_{\lambda}$ let $R$ a rotation in $\mathbf{R}^{N+1}$ that leaves the $x_{N}$-axis fixed and such that

$$
R y=\left(\lambda, 0, \ldots, 0, y_{N}\right) \in \mathbf{R}^{N} .
$$

We then define

$$
\phi_{\lambda, r, y}(x)=\phi_{\lambda, r, R y}(R x) .
$$

Therefore for all $y \in \mathcal{C}_{\lambda}, \phi_{\lambda, r, y}$ is defined for $x \in B_{\lambda, r}(y)$.

Conversely, let $y=\left(\lambda, 0, \ldots, 0, y_{N}\right) \in \mathcal{C}$ and for $x \in \mathbf{R}^{N}$ with $|x|<r$ let

$$
\phi_{\lambda, r, y}^{-1}(x)=\left(\sqrt{\lambda^{2}-\left(x_{1}^{2}+\cdots+x_{N-1}^{2}\right)}, x_{1}, \ldots, x_{N-1}, x_{N}+y_{N}\right) \in B_{\lambda, r}(y) .
$$

Again, for arbitrary $y \in \mathcal{C}$ let $R$ be a rotation in $\mathbf{R}^{N+1}$ that leaves the $x_{N}$-axis (in $\mathbf{R}^{N+1}$ ) fixed such that

$$
R y=\left(\lambda, 0, \ldots, 0, y_{N}\right) .
$$

For $x \in B_{r}(0) \subset \mathbf{R}^{N}$, define

$$
\phi_{\lambda, r, y}^{-1}(x)=R^{-1} \phi_{\lambda, r, R y}^{-1}(x) \in \mathcal{C}_{\lambda} .
$$


At least in the case $y=\left(\lambda, 0, \ldots, 0, y_{N}\right)$ it is clear that $\phi_{\lambda, r, y}$ and $\phi_{\lambda, r, y}^{-1}$ are inverse to each other and are diffeomorphisms between $B_{\lambda, r}(y)$ and $B_{r}(0)$. If $y$ is not contained in the plane $\left(y_{0}, y_{N}\right)$, there is an ambiguity due to nonuniqueness of the rotation $R$. As long as the same $R$ is used in the construction of $\phi_{\lambda, r, y}$ and $\phi_{\lambda, r, y}^{-1}$, the two functions remain inverse to each other. We note here that the Jacobians $J_{\phi_{\lambda, r, y}}(x)$, and $J_{\phi_{\lambda, r, y}^{-1}}(x)$, tend to 1 uniformly on $B_{\lambda, r}(y)$, respectively $B_{r}(0)$, as $r / \lambda \rightarrow 0$.

For $r<\lambda$ and $y \in \mathcal{C}_{\lambda}$ we construct the operators

$$
T_{\lambda, r, y}: H^{1}\left(B_{\lambda, r}(y)\right) \rightarrow H^{1}\left(B_{r}(0)\right) \quad \text { and } \quad \bar{T}_{\lambda, r, y}: H^{1}\left(B_{r}(0)\right) \rightarrow H^{1}\left(B_{\lambda, r}(y)\right),
$$

as follows

$$
T_{\lambda, r, y}(v)(x)=v\left(\phi_{\lambda, r, y}^{-1}(x)\right) \quad \text { and } \quad \bar{T}_{\lambda, r, y}(u)(x)=u\left(\phi_{\lambda, r, y}(x)\right) .
$$

\section{Concentration-compactness}

We need a concentration-compactness lemma which is a more detailed version of that in [21]. By a proof similar to Lemma 4.1 and Lemma 4.2 in [27] we have

LEMmA 3.1. - Let $\lambda_{n} \rightarrow \infty$ as $n \rightarrow \infty$. Let $v_{n} \in H^{1}\left(\mathcal{C}_{\lambda_{n}}\right)$ be such that $I_{\lambda_{n}}\left(v_{n}\right)$ are uniformly bounded and $\int_{\mathcal{C}_{\lambda_{n}}}\left|v_{n}\right|^{p} \mathrm{~d} \mu=1$. Then there is a subsequence (still denoted by $\left.\left(v_{n}\right)\right)$, a nonnegative nonincreasing sequence $\left(\alpha_{i}\right)$ satisfying $\lim _{s \rightarrow \infty} \sum_{i=1}^{s} \alpha_{i}=1$, and sequences $\left(y_{n, i}\right) \subset \mathcal{C}_{\lambda_{n}}$ associated with each $\alpha_{i}>0$ satisfying

$$
\liminf _{n \rightarrow \infty}\left|y_{n, i}-y_{n, j}\right|=\infty, \quad \text { for any } i \neq j,
$$

such that the following property holds: If $\alpha_{s}>0$ for some $s \geqslant 1$, then for $\varepsilon>0$ there exist $R>0$, for all $r \geqslant R$ and all $r^{\prime} \geqslant R$

$$
\left.\limsup _{n \rightarrow \infty} \sum_{i=1}^{s}\left|\alpha_{i}-\int_{B_{\lambda_{n}, r}\left(y_{n, i}\right)}\right| v_{n}\right|^{p} \mathrm{~d} \mu|+|\left(1-\sum_{i=1}^{s} \alpha_{i}\right)-\int_{\mathcal{C}_{\lambda_{n}} \backslash \bigcup_{i=1}^{s} B_{\lambda_{n}, r^{\prime}}\left(y_{n, i}\right)}\left|v_{n}\right|^{p} \mathrm{~d} \mu \mid<\varepsilon .
$$

Proposition 3.2. - For a subsequence as in Lemma 3.1, we have that for $s \geqslant 1$,

$$
\liminf _{n \rightarrow \infty} I_{\lambda_{n}}\left(v_{n}\right) \geqslant S_{p}\left(\mathbf{R}^{N}\right)\left(\sum_{i=1}^{s} \alpha_{i}^{2 / p}+\left(1-\sum_{i=1}^{s} \alpha_{i}\right)^{2 / p}\right) .
$$

Consequently,

$$
\liminf _{n \rightarrow \infty} I_{\lambda_{n}}\left(v_{n}\right) \geqslant S_{p}\left(\mathbf{R}^{N}\right) \sum_{i=1}^{\infty} \alpha_{i}^{2 / p}
$$

Proof. - Denote

$$
S_{p}\left(\mathcal{C}_{\lambda}\right)=\inf _{v \in H^{1}\left(\mathcal{C}_{\lambda}\right) \backslash\{0\}} I_{\lambda}(v) .
$$


It is not difficult to check (see the proof of Theorem 1.2 in [10]) that

$$
\lim _{\lambda \rightarrow \infty} S_{p}\left(\mathcal{C}_{\lambda}\right)=S_{p}\left(\mathbf{R}^{N}\right)
$$

Fix $s$, and assume

$$
\sum_{i=1}^{s} \alpha_{i}<1
$$

We choose

$$
0<\varepsilon<\frac{1}{2} \min \left\{\alpha_{s}, 1-\sum_{i=1}^{s} \alpha_{i}\right\},
$$

and the corresponding $R$ as given in Lemma 3.1. Let $r \geqslant R$ and for $n$ large we can assume that $B_{\lambda_{n}, 3 r}\left(y_{n, i}\right)$ are disjoint for $i=1, \ldots, s$, and

$$
\begin{gathered}
\int_{B_{\lambda_{n}, r}\left(y_{n, i}\right)}\left|v_{n}\right|^{p} \mathrm{~d} \mu \geqslant \alpha_{i}-2 \varepsilon, \\
\int_{\mathcal{C}_{\lambda_{n}} \backslash \bigcup_{i=1}^{s} B_{\lambda_{n}, 3 r}\left(y_{n, i}\right)}\left|v_{n}\right|^{p} \mathrm{~d} \mu \geqslant\left(1-\sum_{i=1}^{s} \alpha_{i}\right)-2 \varepsilon .
\end{gathered}
$$

For $i=1, \ldots, s$, let $0 \leqslant \rho_{n, i} \leqslant 1$ cut-off functions with $\left|\nabla \rho_{n, i}\right|(x) \leqslant 2 / r$, which are identically 1 inside $B_{\lambda_{n}, r}\left(y_{n, i}\right)$ and 0 outside $B_{\lambda_{n}, 2 r}\left(y_{n, i}\right)$. We also define $0 \leqslant \rho_{n} \leqslant 1$ a cut-off function on $\mathcal{C}_{\lambda_{n}}$ such that $\rho_{n}$ is identically 1 on $\mathcal{C}_{\lambda_{n}} \backslash \bigcup_{i=1}^{s} B_{\lambda_{n}, 3 r}\left(y_{n, i}\right)$ and 0 on $\bigcup_{i=1}^{s} B_{\lambda_{n}, 2 r}\left(y_{n, i}\right)$, with $\left|\nabla \rho_{n}\right|(x) \leqslant 2 / r$. Let

$$
w_{n, i}(x)=\rho_{n, i}(x) v_{n}(x) \quad \text { for } x \in \mathcal{C}_{\lambda_{n}} .
$$

Also, define $w_{n}=\rho_{n} v_{n}$. Then

$$
\int_{\mathcal{C}_{\lambda_{n}}}\left|w_{n, i}\right|^{p} \mathrm{~d} \mu \geqslant \int_{B_{\lambda_{n}, r}\left(y_{n, i}\right)}\left|v_{n}\right|^{p} \mathrm{~d} \mu,
$$

and

$$
\int_{\mathcal{C}_{\lambda_{n}}}\left|w_{n}\right|^{p} \mathrm{~d} \mu \geqslant \int_{\mathcal{C}_{\lambda_{n}} \backslash \bigcup_{i=1}^{s} B_{\lambda_{n}, 3 r}\left(y_{n, i}\right)}\left|v_{n}\right|^{p} \mathrm{~d} \mu .
$$

From (13), (15), and (14), (16), we obtain

$$
\int_{\mathcal{C}_{\lambda_{n}}}\left|\nabla w_{n, i}\right|^{2}+w_{n, i}^{2} \mathrm{~d} \mu \geqslant S_{p}\left(\mathcal{C}_{\lambda_{n}}\right)\left(\alpha_{i}-2 \varepsilon\right)^{2 / p},
$$


and

$$
\int_{\mathcal{C}_{\lambda_{n}}}\left|\nabla w_{n}\right|^{2}+w_{n}^{2} \mathrm{~d} \mu \geqslant S_{p}\left(\mathcal{C}_{\lambda_{n}}\right)\left(1-\sum_{i=1}^{s} \alpha_{i}-2 \varepsilon\right)^{2 / p}
$$

Using the fact that the Jacobians $J_{\phi_{\lambda, r, y_{n, i}}}(x)$ and $J_{\phi_{\lambda, r, y_{n, i}}^{-1}}(x)$ tend to 1 uniformly on $B_{\lambda, r}\left(y_{n, i}\right)$, respectively $B_{r}(0)$, as $\lambda \rightarrow \infty$, we also have

$$
\int_{B_{\lambda_{n}, 2 r}\left(y_{n, i}\right)}\left|\nabla v_{n}\right|^{2}+v_{n}^{2} \mathrm{~d} \mu \geqslant \int_{\mathcal{C}_{\lambda_{n}}}\left|\nabla w_{n, i}\right|^{2}+w_{n, i}^{2} \mathrm{~d} \mu-\frac{C}{r^{2}},
$$

and

$$
\int_{\mathcal{C}_{\lambda_{n}} \backslash \bigcup_{i=1}^{s} B_{\lambda_{n}, 2 r}\left(y_{n, i}\right)}\left|\nabla v_{n}\right|^{2}+v_{n}^{2} \mathrm{~d} \mu \geqslant \int_{\mathcal{C}_{\lambda_{n}}}\left|\nabla w_{n}\right|^{2}+w_{n}^{2} \mathrm{~d} \mu-\frac{C}{r^{2}},
$$

with $C$ independent of $r$ and $n$. Therefore,

$$
I_{\lambda_{n}}\left(v_{n}\right) \geqslant S_{p}\left(\mathcal{C}_{\lambda_{n}}\right)\left(\sum_{i=1}^{s}\left(\alpha_{i}-2 \varepsilon\right)^{2 / p}+\left(\left(1-\sum_{i=1}^{s} \alpha_{i}\right)-2 \varepsilon\right)^{2 / p}\right)-\frac{(s+1) C}{r^{2}} .
$$

Let $n \rightarrow \infty$, then $r \rightarrow \infty$, and finally $\varepsilon \rightarrow 0$. The case $\sum_{i=1}^{s} \alpha_{i}=1$ is done the same way, without considering $w_{n}$ anymore.

\section{Main results for Eq. (8)}

In this section we shall state and prove our results for Eq. (8) on $\mathcal{C}_{\lambda}$. These results, which are of their own interest, will in turn give our main results for Eq. (1) by using the transformation (5).

It is known (e.g., [14,18,29]) that $S_{p}\left(\mathbf{R}^{N}\right)$ is achieved by a positive function $U \in$ $H^{1}\left(\mathbf{R}^{N}\right)$ such that $|U|_{L^{p}}=1$ and

$$
\int_{\mathbf{R}^{N}}|\nabla U|^{2}+U^{2} \mathrm{~d} x=S_{p}\left(\mathbf{R}^{N}\right)
$$

Moreover, $U$ is unique up to translations and it is radially symmetric with respect to some point (which we assume to be the origin in $\mathbf{R}^{N}$ ). We place $\mathcal{C}_{\lambda}$ in $\mathbf{R}^{N+1}$ as in (9). $\mathcal{C}_{\lambda}$ is invariant under the canonical action of $\mathbf{O}(N) \times \mathbf{O}(1) \subset \mathbf{O}(N+1)$ with $\mathbf{O}(N)$ acting on $\mathbf{R}^{N}=\left\{x \in \mathbf{R}^{N+1} \mid x_{N}=0\right\}$ and $\mathbf{O}(1)$ acting on $\mathbf{R}=\left\{x \in \mathbf{R}^{N+1} \mid x_{0}=\cdots=x_{N-1}=0\right\}$. Eq. (8) is also invariant under this group. For a function $v \in H^{1}\left(\mathcal{C}_{\lambda}\right)$ we define the isotropy subgroup of $v$ as

$$
\Sigma_{v}=\{g \in \mathbf{O}(N) \times \mathbf{O}(1) \mid g v=v\} .
$$


Here $g v(x)=v\left(g^{-1} x\right)$. In the following we denote by $\tilde{G}$ a subgroup in $\mathbf{O}(N) \times \mathbf{O}(1)$, given by

$$
\tilde{G}=G \times \mathbf{O}(1)
$$

with $G$ a subgroup of $\mathbf{O}(N)$. We shall prove

THEOREM 4.1. - Let $\Omega \subset \mathbf{S}^{N-1} \times\{0\} \subset \mathcal{C}$ be a locally minimal orbit set of $G$ with the corresponding integer $k \in \mathbf{N}$. Then for $\lambda$ sufficiently large, problem (8) has a solution $w_{\lambda}$ satisfying

(i) $w_{\lambda}$ is $\tilde{G}$-invariant,

(ii) $w_{\lambda}$ has exactly $k$ maximum points which form a $\tilde{G}$ orbit $\tilde{G} y_{\lambda}$ for some $y_{\lambda} \in \Omega_{\lambda}:=$ $\lambda \Omega$,

(iii) $w_{\lambda}$ is of $k$-bump type in the sense that if $\left\{y_{\lambda, 1}, \ldots, y_{\lambda, k}\right\}$ are the maxima of $w_{\lambda}$, then

$$
\lim _{\lambda \rightarrow \infty}\left\|w_{\lambda}-\sum_{i=1}^{k} \bar{T}_{\lambda, \sqrt{\lambda}, y_{\lambda, i}}\left(S_{p}\left(\mathbf{R}^{N}\right)^{1 /(p-2)} U\right)\right\|_{H^{1}\left(\mathcal{C}_{\lambda}\right)}=0
$$

(iv) $\lim _{\lambda \rightarrow \infty} I_{\lambda}\left(w_{\lambda}\right)=k^{(p-2) / p} S_{p}\left(\mathbf{R}^{N}\right)$,

(v) if in addition, $G$ is maximal with respect to $\Omega$, then $\Sigma_{w_{\lambda}}=\tilde{G}$.

The idea of proving this result stems out of our work in [8] and [27] where we have given a rather general local minimization scheme to construct positive solutions with prescribed symmetry for some radially invariant elliptic problems. The procedure used below is somewhat different from that in [8] and [27] and seems to be more transparent. We set up our minimization scheme around $\Omega$. Since $\Omega$ is a locally minimal orbit set, there is $\delta>0$ such that

$$
\# G y>k \text { for all } y \in \mathbf{S}^{N-1} \times\{0\} \text { with } 0<\operatorname{dist}(y, \Omega)<3 \delta \text {. }
$$

Denote

$$
\Lambda=\{y \in \mathcal{C}: \operatorname{dist}(y, \Omega)<\delta\} \quad \text { and } \quad \Pi=\{y \in \mathcal{C}: \operatorname{dist}(y, \Omega)<2 \delta\}
$$

Note that because $G$ is a group of isometries, the sets $\Lambda$ and $\Pi$ are invariant under the action of $G$.

Since we work on cylinders of different radii, we make the following notations $\Omega_{\lambda}:=\lambda \Omega, \Lambda_{\lambda}:=\lambda \Lambda$, and $\Pi_{\lambda}:=\lambda \Pi$, all subsets of $\mathcal{C}_{\lambda}$.

In the space of $\tilde{G}$-symmetric functions

$$
\mathcal{H}_{G, \lambda}=\left\{v \in H^{1}\left(\mathcal{C}_{\lambda}\right): g v=v, \text { for all } g \in \tilde{G}\right\},
$$

we define a constraint manifold and an open subset of it

$$
\mathcal{M}_{G, \lambda}=\left\{v \in \mathcal{H}_{G, \lambda}: \int_{\mathcal{C}_{\lambda}}|v|^{p}=1\right\}
$$




$$
\mathcal{K}_{G, \lambda}^{\sigma}=\left\{u \in \mathcal{M}_{G, \lambda}: \gamma_{\lambda}(v)>1-\frac{\sigma}{2}\right\},
$$

where

$$
\gamma_{\lambda}(v)=\int_{\Lambda_{\lambda}}|v|^{p} \mathrm{~d} \mu \quad \text { and } \quad \sigma=\frac{1}{k+1} .
$$

Note that $\gamma_{\lambda}$ is a continuous functional on $H^{1}\left(\mathcal{C}_{\lambda}\right)$ and $\mathcal{K}_{G, \lambda}^{\sigma}$ is an open subset of $\mathcal{M}_{G, \lambda}$. We are looking for critical points of the functional

$$
I_{\lambda}(v)=\int_{C_{\lambda}}|\nabla v|^{2}+v^{2} \mathrm{~d} \mu, \quad \text { with } v \in \mathcal{K}_{G, \lambda}^{\sigma} .
$$

Provided $\lambda$ is large, we show that

$$
m_{\lambda, \sigma}:=\inf _{u \in \mathcal{K}_{G, \lambda}^{\sigma}} I_{\lambda}(u)
$$

is achieved by a function in the interior of $\mathcal{K}_{G, \lambda}^{\sigma}$ (which can be assumed to be nonnegative). By [25], a critical point in $\mathcal{M}_{G, \lambda}$ of $I_{\lambda}$, after a rescaling will be a solution of (8).

First, we have the following local compactness result.

THEOREM 4.2. - Let $\lambda>0$ fixed. If there is a minimizing sequence $\left(v_{m}\right) \subset \mathcal{K}_{G, \lambda}^{\sigma}$ (i.e. $\left.I_{\lambda}\left(v_{m}\right) \rightarrow m_{\lambda, \sigma}\right)$ such that for $m$ large

$$
\gamma_{\lambda}\left(v_{m}\right) \geqslant \frac{m_{\lambda, \sigma}^{p /(p-2)}}{S_{p}\left(\mathcal{C}_{\lambda}\right)^{p /(p-2)}+m_{\lambda, \sigma}^{p /(p-2)}},
$$

then there is a subsequence (still denoted $\left(v_{m}\right)$ ) which converges strongly to $v \in \overline{\mathcal{K}}_{G, \lambda}^{\sigma}$ (the closure in $\left.\mathcal{H}_{G, \lambda}\right)$, and $I_{\lambda}(v)=m_{\lambda, \sigma}$.

Proof. - Since $\left(v_{m}\right)$ is a minimizing sequence, it is bounded in $H^{1}\left(\mathcal{C}_{\lambda}\right)$. Therefore we can assume

$$
\begin{array}{lll}
\text { (i) } & v_{m} \rightarrow v & \text { in } H^{1}\left(\mathcal{C}_{\lambda}\right) \\
\text { (ii) } & v_{m} \rightarrow v & \text { in } L_{\operatorname{loc}}^{p}\left(\mathcal{C}_{\lambda}\right) \\
\text { (iii) } & v_{m} \rightarrow v & \text { a.e. in } \mathcal{C}_{\lambda} .
\end{array}
$$

Since $\bar{\Lambda}$ is compact, it follows from (ii) that

$$
\int_{\Lambda}|v|^{p} \mathrm{~d} \mu \geqslant 1-\frac{\sigma}{2}
$$

therefore $v \not \equiv 0$. If

$$
\int_{\mathcal{C}_{\lambda}}|v|^{p} \mathrm{~d} \mu=1
$$


170 F. CATRINA, Z.-Q. WANG / Ann. Inst. Henri Poincaré, Anal. non linéaire 18 (2001) 157-178 then $v \in \overline{\mathcal{K}}_{G, \lambda}^{\sigma}$, and since $I_{\lambda}$ is lower semi-continuous, it follows that

$$
I_{\lambda}(v) \leqslant m_{\lambda, \sigma},
$$

hence $I_{\lambda}(v)=m_{\lambda, \sigma}$.

Therefore, assume that $\int_{\mathcal{C}_{\lambda}}|v|^{p} \mathrm{~d} \mu<1$. This implies

$$
\frac{v}{|v|_{L^{p}\left(\mathcal{C}_{\lambda}\right)}^{2}} \in \mathcal{K}_{G, \lambda}^{\sigma}, \quad \text { hence } \int_{\mathcal{C}_{\lambda}}|\nabla v|^{2}+v^{2} \mathrm{~d} \mu \geqslant m_{\lambda, \sigma}\left(\int_{\mathcal{C}_{\lambda}}|v|^{p} \mathrm{~d} \mu\right)^{2 / p}
$$

We have

$$
\begin{aligned}
m_{\lambda, \sigma} & =\lim _{m \rightarrow \infty} \int_{\mathcal{C}_{\lambda}}\left|\nabla v_{m}\right|^{2}+v_{m}^{2} \mathrm{~d} \mu \\
& =\int_{\mathcal{C}_{\lambda}}|\nabla v|^{2}+v^{2} \mathrm{~d} \mu+\lim _{m \rightarrow \infty} \int_{\mathcal{C}_{\lambda}}\left|\nabla\left(v_{m}-v\right)\right|^{2}+\left(v_{m}-v\right)^{2} \mathrm{~d} \mu \\
& \geqslant \int_{\mathcal{C}_{\lambda}}|\nabla v|^{2}+v^{2} \mathrm{~d} \mu+S_{p}\left(\mathcal{C}_{\lambda}\right) \lim _{m \rightarrow \infty}\left(\int_{\mathcal{C}_{\lambda}}\left|v_{m}-v\right|^{p} \mathrm{~d} \mu\right)^{2 / p} .
\end{aligned}
$$

Therefore,

$$
\int_{\mathcal{C}_{\lambda}}|\nabla v|^{2}+v^{2} \mathrm{~d} \mu \leqslant m_{\lambda, \sigma}-S_{p}\left(\mathcal{C}_{\lambda}\right) \lim _{m \rightarrow \infty}\left(\int_{\mathcal{C}_{\lambda}}\left|v_{m}-v\right|^{p} \mathrm{~d} \mu\right)^{2 / p} .
$$

On the other hand, from the Brezis-Lieb lemma (see [3]),

$$
1=\lim _{m \rightarrow \infty} \int_{\mathcal{C}_{\lambda}}\left|v_{m}\right|^{p} \mathrm{~d} \mu=\int_{\mathcal{C}_{\lambda}}|v|^{p} \mathrm{~d} \mu+\lim _{m \rightarrow \infty} \int_{\mathcal{C}_{\lambda}}\left|v_{m}-v\right|^{p} \mathrm{~d} \mu .
$$

Hence

$$
\lim _{m \rightarrow \infty} \int_{\mathcal{C}_{\lambda}}\left|v_{m}-v\right|^{p} \mathrm{~d} \mu=1-\int_{\mathcal{C}_{\lambda}}|v|^{p} \mathrm{~d} \mu
$$

From (20) and (21), we obtain

$$
\int_{\mathcal{C}_{\lambda}}|\nabla v|^{2}+v^{2} \mathrm{~d} \mu \leqslant m_{\lambda, \sigma}-S_{p}\left(\mathcal{C}_{\lambda}\right)\left(1-\int_{\mathcal{C}_{\lambda}}|v|^{p} \mathrm{~d} \mu\right)^{2 / p} .
$$

Again from (ii) and the hypothesis we have

$$
1>x:=\int_{\mathcal{C}_{\lambda}} v^{p} \mathrm{~d} \mu \geqslant \frac{m_{\lambda, \sigma}^{p /(p-2)}}{S_{p}\left(\mathcal{C}_{\lambda}\right)^{p /(p-2)}+m_{\lambda, \sigma}^{p /(p-2)}}
$$


From (19) and (22), we obtain

$$
0 \leqslant m_{\lambda, \sigma}\left(1-x^{2 / p}\right)-S_{p}\left(\mathcal{C}_{\lambda}\right)(1-x)^{2 / p} .
$$

A direct check shows that (23) is not possible.

Next, we need some asymptotic estimates for $\lambda$ large. For notations convenience, we let for $\lambda_{n} \rightarrow \infty$

$$
\lambda_{n} \Omega=\Omega_{n} \subset \Lambda_{n} \subset \Pi_{n} \subset \mathcal{C}_{\lambda_{n}} .
$$

PROPOSITION 4.3. - We have

$$
\limsup _{\lambda \rightarrow \infty} m_{\lambda, \sigma} \leqslant k^{(p-2) / p} S_{p}\left(\mathbf{R}^{N}\right) .
$$

Proof. - We prove the proposition by constructing test functions. Let $U \in H^{1}\left(\mathbf{R}^{N}\right)$ such that $|U|_{L^{p}}=1$ and

$$
\int_{\mathbf{R}^{N}}|\nabla U|^{2}+U^{2} \mathrm{~d} x=S_{p}\left(\mathbf{R}^{N}\right) .
$$

For an $\varepsilon>0$, let $r>0$ sufficiently large so that there is a radial cut-off function $\rho(x)$ identically 1 in $B_{r}(0)$ and 0 outside $B_{2 r}(0)$, such that the function $u(x)=\rho(x) U(x)$ has the property

$$
\frac{\int_{\mathbf{R}^{N}}|\nabla u|^{2}+u^{2} \mathrm{~d} x}{|u|_{L^{p}\left(\mathbf{R}^{N}\right)}^{2}} \leqslant S_{p}\left(\mathbf{R}^{N}\right)+\varepsilon .
$$

For a point $y_{1} \in \Omega_{\lambda}$ let us denote $\left\{y_{1}, \ldots, y_{k}\right\}=\tilde{G} y_{1}$. Consider $\lambda$ sufficiently large so that $B_{\lambda, 2 r}\left(y_{i}\right)$ are disjoint and contained in $\Lambda_{\lambda}$. Let

$$
v_{i}=\bar{T}_{\lambda, 2 r, y_{i}} u,
$$

and consider $v \in H^{1}\left(\mathcal{C}_{\lambda}\right)$ given by

$$
v=\sum_{i=1}^{k} \frac{v_{i}}{k^{1 / p}\left|v_{i}\right|_{L^{p}}} .
$$

Clearly, $v \in \mathcal{K}_{G, \lambda}^{\sigma}$ for $\lambda$ large. We obtain

$$
m_{\lambda, \sigma} \leqslant I_{\lambda}(v)=k^{(p-2) / p} I_{\lambda}\left(v_{1}\right) .
$$

Therefore,

$$
\limsup _{\lambda \rightarrow \infty} m_{\lambda, \sigma} \leqslant k^{(p-2) / p} \frac{\int_{\mathbf{R}^{N}}|\nabla u|^{2}+u^{2} \mathrm{~d} x}{|u|_{L^{p}}^{2}} \leqslant k^{(p-2) / p} S_{p}\left(\mathbf{R}^{N}\right)+\varepsilon .
$$

Let $\varepsilon \rightarrow 0$. 
172 F. CATRINA, Z.-Q. WANG / Ann. Inst. Henri Poincaré, Anal. non linéaire 18 (2001) 157-178

Proposition 4.4. - For any sequences $\lambda_{n} \rightarrow \infty$ and $v_{n} \in \mathcal{K}_{G, \lambda_{n}}^{\sigma}$ such that

$$
\lim _{n \rightarrow \infty} I_{\lambda_{n}}\left(v_{n}\right) \rightarrow d \leqslant k^{(p-2) / p} S_{p}\left(\mathbf{R}^{N}\right)
$$

we have

$$
\int_{\Lambda_{n}} v_{n}^{p} \mathrm{~d} \mu \rightarrow 1 \quad \text { and } \quad d=k^{(p-2) / p} S_{p}\left(\mathbf{R}^{N}\right) .
$$

To prove this proposition, we need a lemma.

LEMMA 4.5. - Applying Lemma 3.1 to a sequence $v_{n} \in \mathcal{K}_{G, \lambda_{n}}^{\sigma}$ we get the sequences $\left(\alpha_{i}\right),\left(y_{n, i}\right)$ such that (10) and (11) hold. Then we have either $\operatorname{dist}\left(y_{n, i}, \Lambda_{n}\right) \rightarrow \infty$ as $n \rightarrow \infty$, or there exists an $n_{i}$ so that for $n \geqslant n_{i}$ we have $y_{n, i} \in \Pi_{n}$.

Proof. - According to Observation 2.6 and Proposition 2.8 in [8], we can assume that for any $1 \leqslant i \leqslant s$ fixed, there is an orthonormal frame $\left(\xi_{1}^{i}, \ldots, \xi_{t_{i}}^{i}\right)$ in $\mathbf{R}^{N+1}$, independent of $n$, such that

$$
y_{n, i}=b_{n, 1}^{i} \xi_{1}^{i}+\cdots+b_{n, t_{i}}^{i} \xi_{t_{i}}^{i}
$$

where

$$
b_{n, j}^{i} \rightarrow \infty \text { as } n \rightarrow \infty, \text { for } j=1, \ldots, t_{i},
$$

and

$$
\frac{b_{n, j+1}^{i}}{b_{n, j}^{i}} \rightarrow 0 \quad \text { as } n \rightarrow \infty, \text { for } j=1, \ldots, t_{i}-1 .
$$

If the direction of $\xi_{1}^{i}$ intersects $\mathcal{C}$ at a point $\eta$ in the closure $\bar{\Lambda}$, then

$$
|\eta| \frac{y_{n, i}}{\lambda_{n}} \rightarrow \eta, \quad \text { as } n \rightarrow \infty
$$

Therefore, if $n$ is sufficiently large, $y_{n} \in \Pi_{n}$.

If $\operatorname{dist}(\eta, \Lambda)=d>0$, then

$$
\operatorname{dist}\left(y_{n, i}, \Lambda_{n}\right)=d \lambda_{n}+\mathrm{O}\left(b_{n, 2}^{i}\right)
$$

which tends to infinity.

Proof of Proposition 4.4. - Applying Lemma 3.1 to the sequence $\left(v_{n}\right)$ we get the sequences $\left(\alpha_{i}\right),\left(y_{n, i}\right)$ such that (10) and (11) hold. Let $s$ be such that $\alpha_{s}>\alpha_{s+1}$ and

$$
\sum_{i=1}^{s} \alpha_{i}>1-\frac{\sigma}{2}
$$

Choose

$$
0<\varepsilon<\min \left\{\frac{\alpha_{i}-\alpha_{i+1}}{2}: \alpha_{i} \neq \alpha_{i+1}, i=1, \ldots, s\right\}
$$


From Lemma 4.5 , by relabeling $\alpha_{1}, \ldots, \alpha_{s}$ we can assume that directions $\xi_{1}^{1}, \ldots, \xi_{1}^{s_{1}}$ intersect $\mathcal{C}$ in $\bar{\Lambda}$. Hence, for $n$ bigger than $\max \left\{n_{i}: i=1, \ldots, s_{1}\right\}$ (see Lemma 4.5), $y_{n, i} \in \Pi_{n}$ for $i=1, \ldots, s_{1}$. Also, $\operatorname{dist}\left(y_{n, i}, \Lambda_{n}\right) \rightarrow \infty$ for $i=s_{1}+1, \ldots, s$. We show that

$$
\sum_{i=1}^{s_{1}} \alpha_{i} \geqslant 1-\sigma
$$

Indeed, from Lemma 3.1, for $n$ large we have

$$
\sum_{i=1}^{s_{1}} \alpha_{i} \geqslant \sum_{i=1}^{s_{1}} \int_{B_{\lambda_{n}, r}\left(y_{n, i}\right)}\left|v_{n}\right|^{p} \mathrm{~d} \mu-2 \varepsilon
$$

and from (24) we get

$$
\int_{\Lambda_{n} \backslash \bigcup_{i=1}^{s_{1}} B_{\lambda_{n}, r}\left(y_{n, i}\right)}\left|v_{n}\right|^{p} \mathrm{~d} \mu-\frac{\sigma}{2}-\varepsilon<0 .
$$

Therefore,

$$
\sum_{i=1}^{s_{1}} \alpha_{i} \geqslant \int_{\Lambda_{n}}\left|v_{n}\right|^{p} \mathrm{~d} \mu-\frac{\sigma}{2}-3 \varepsilon
$$

Since

$$
\int_{\Lambda_{n}}\left|v_{n}\right|^{p} \mathrm{~d} \mu>1-\frac{\sigma}{2}
$$

let $n \rightarrow \infty$ and then $\varepsilon \rightarrow 0$; (25) follows. Without loss of generality, we can assume that for all $n, y_{n, i}$ appear in orbits. I.e. if $y_{n, i}$ corresponds to $\alpha_{i}$ and $g \in \tilde{G}$, then $g y_{n, i}$ is an $y_{n, j}$ corresponding to some $\alpha_{j}=\alpha_{i}$ (because of symmetry). We denote

$$
\left\{\alpha_{i}: i=1, \ldots, s\right\} / \tilde{G}=\left\{\bar{\alpha}_{\iota}: \iota=1, \ldots, \bar{s}\right\}
$$

where $\alpha_{i}$ is in the same class with $\alpha_{j}$ if $y_{n, i}$ and $y_{n, j}$ are in the same orbit. We also denote $k_{\iota}$ the number of elements in the class $\bar{\alpha}_{\iota}$ for $\iota=1, \ldots, \bar{s}$, i.e. $k_{\iota}=\# \tilde{G} y_{n, i}$ with $\alpha_{i}$ in the class $\bar{\alpha}_{\iota}$. From Proposition 3.2, we now have

$$
I_{\lambda_{n}}\left(v_{n}\right) \geqslant \sum_{\imath=1}^{\bar{s}_{1}} k_{\iota} \bar{\alpha}_{\iota}^{2 / p} S_{p}\left(\mathbf{R}^{N}\right)+\left(1-\sum_{i=1}^{s_{1}} \alpha_{i}\right)^{2 / p} S_{p}\left(\mathbf{R}^{N}\right)-\mathrm{o}(1),
$$

with o(1) $\rightarrow 0$ as $n \rightarrow \infty$. Let $n \rightarrow \infty$ and we get

$$
k^{(p-2) / p} S_{p}\left(\mathbf{R}^{N}\right) \geqslant d \geqslant S_{p}\left(\mathbf{R}^{N}\right)\left(\sum_{\iota=1}^{\bar{s}_{1}} k_{\iota} \bar{\alpha}_{\iota}^{2 / p}+\left(1-\sum_{\iota=1}^{\bar{s}_{1}} k_{\iota} \bar{\alpha}_{\iota}\right)^{2 / p}\right) .
$$


174 F. CATRINA, Z.-Q. WANG / Ann. Inst. Henri Poincaré, Anal. non linéaire 18 (2001) 157-178

Since $k_{\iota} \geqslant k$, we have

$$
\sum_{\iota=1}^{\bar{s}_{1}} k_{\iota} \bar{\alpha}_{\iota}^{2 / p}+\left(1-\sum_{\iota=1}^{\bar{s}_{1}} k_{\iota} \bar{\alpha}_{\iota}\right)^{2 / p} \geqslant k^{(p-2) / 2} x^{2 / p}+(1-x)^{2 / p}
$$

where

$$
x=\sum_{\iota=1}^{\bar{s}_{1}} k_{\iota} \bar{\alpha}_{\iota}
$$

Because $\sigma=1 /(k+1)$ and (25), we have $x \geqslant k /(k+1)$, and this implies

$$
k^{(p-2) / 2} x^{2 / p}+(1-x)^{2 / p} \geqslant k^{(p-2) / 2},
$$

with equality if and only if $x=1$. From (26) we see that we need equality, and the only way this can happen is if $s_{1}=k, y_{n, 1} \in \Omega_{n}$, and $\sum_{i=1}^{k} \alpha_{i}=1$.

Now we are ready to give the proof of Theorem 4.1 .

Proof of Theorem 4.1. - We argue that there is $\lambda_{k}>0$ such that $\lambda \geqslant \lambda_{k}$, and $v \in \mathcal{K}_{G, \lambda}^{\sigma}$ with $I_{\lambda}(v)<m_{\lambda, \sigma}+\frac{1}{\lambda}$, implies

$$
\gamma_{\lambda}(v)>\max \left\{1-\frac{\sigma}{4}, \frac{m_{\lambda, \sigma}^{p /(p-2)}}{S_{p}\left(\mathcal{C}_{\lambda}\right)^{p /(p-2)}+m_{\lambda, \sigma}^{p /(p-2)}}\right\} .
$$

Indeed, if the statement above is false, there are $\lambda_{n} \rightarrow \infty$, and $v_{n} \in \mathcal{K}_{G, \lambda_{n}}^{\sigma}$, such that $I_{\lambda_{n}}\left(v_{n}\right)<m_{\lambda_{n}, \sigma}+\frac{1}{\lambda_{n}}$ and

$$
\gamma_{\lambda_{n}}\left(v_{n}\right) \leqslant \max \left\{1-\frac{\sigma}{4}, \frac{m_{\lambda_{n}, \sigma}^{p /(p-2)}}{S_{p}\left(\mathcal{C}_{\lambda_{n}}\right)^{p /(p-2)}+m_{\lambda_{n}, \sigma}^{p /(p-2)}}\right\} .
$$

By Propositions 4.3 and 4.4 we have

$$
\lim _{\lambda_{n} \rightarrow \infty} m_{\lambda_{n}, \sigma} \rightarrow k^{(p-2) / p} S_{p}\left(\mathbf{R}^{N}\right)
$$

and again by Proposition 4.4 we obtain,

$$
\int_{\Lambda_{\lambda_{n}}}\left|v_{n}\right|^{p} \mathrm{~d} \mu \rightarrow 1
$$

contradicting (27).

Now, by Theorem 4.2, for $\lambda \geqslant \lambda_{k}, m_{\lambda, \sigma}$ is achieved by $v \in \mathcal{K}_{G, \lambda}^{\sigma}$ (because $\gamma_{\lambda}(v)>$ $\left.1-\frac{\sigma}{4}\right)$.

For $v_{\lambda} \in \mathcal{K}_{G, \lambda}^{\sigma}$ critical point of $I_{\lambda}$ with $I_{\lambda}(v)=m_{\lambda, \sigma}$, we define

$$
w_{\lambda}=m_{\lambda, \sigma}^{1 /(p-2)} v_{\lambda}
$$


Then $w_{\lambda}$ is a solution of (8). Note that

$$
\int_{\mathcal{C}_{\lambda}}\left|\nabla w_{\lambda}\right|^{2}+w_{\lambda}^{2} \mathrm{~d} \mu=\int_{\mathcal{C}_{\lambda}} w_{\lambda}^{p} \mathrm{~d} \mu=m_{\lambda, \sigma}^{p /(p-2)} .
$$

Part (i) of Theorem 4.1 follows from the construction of $w_{\lambda}$.

To prove (ii), assume by contradiction that there is a sequence $\lambda_{n} \rightarrow \infty$ such that the corresponding solutions $w_{n}$ of (8) have more than $k$ local maximum points. We shall derive a contradiction with (29).

Eventually by performing a rotation in $\left(x_{0}, x_{1}, \ldots, x_{N-1}\right)$, and a translation in $x_{N}$ for each $n$, we can assume without loss of generality that $y_{n}=\left(\lambda_{n}, 0, \ldots, 0\right)$ is a local maximum of $w_{n}$. Let $r_{n} \rightarrow \infty$ such that $r_{n} / \lambda_{n} \rightarrow 0$ as $n \rightarrow \infty$. We denote

$$
u_{n}=T_{\lambda_{n}, r_{n}, y_{n}}\left(w_{n}\right) \in H^{1}\left(B_{r_{n}}(0)\right) .
$$

Since $w_{n}$ are solutions of (8), we get that $u_{n}$ satisfies

$$
-\sum_{i, j=1}^{N-1} a_{i j}^{n}(x) \frac{\partial^{2} u_{n}}{\partial x_{i} \partial x_{j}}+\sum_{i=1}^{N-1} b_{i}^{n}(x) \frac{\partial u_{n}}{\partial x_{i}}+u_{n}=u_{n}^{p-1}, \quad \text { in } B_{r_{n}}(0),
$$

where

$$
a_{i j}^{n}(x)=\delta_{i j}-\frac{x_{i} x_{j}}{\lambda_{n}^{2}} \quad \text { and } \quad b_{i}^{n}(x)=(N-1) \frac{x_{i}}{\lambda_{n}^{2}} .
$$

Since $r_{n} / \lambda_{n} \rightarrow 0$, it is easy to check that $a_{i j}^{n}(x) \rightarrow \delta_{i j}$ and $b_{i}^{n}(x) \rightarrow 0$, uniformly in $B_{r_{n}}(0) \subset \mathbf{R}^{N}$. Elliptic theory (see [15]) gives

$$
u_{n} \rightarrow u \quad \text { in } C_{\text {loc }}^{2}\left(\mathbf{R}^{N}\right)
$$

where $u$ is a solution of

$$
-\Delta u+u=u^{p-1} \quad \text { in } \mathbf{R}^{N} .
$$

Since $y_{n}$ is a local maximum for $w_{n}$ and $w_{n}\left(y_{n}\right) \geqslant 1$ for all $n$, it follows $0 \in \mathbf{R}^{N}$ is a local maximum for $u_{n}$, hence for $u$. Therefore, $u$ is a nonzero solution of (31). By Fatou's lemma and since $u_{n} \in H^{1}\left(B_{r_{n}}(0)\right)$ and $u_{n} \geqslant 0$ for all $n$, we have $u \in H^{1}\left(\mathbf{R}^{N}\right)$ and $u \geqslant 0$. The only nonnegative, nonzero solution with its maximum at 0 of $(31)$ in $H^{1}\left(\mathbf{R}^{N}\right)$, is

$$
u(x)=S_{p}\left(\mathbf{R}^{N}\right)^{1 /(p-2)} U(x) .
$$

Since $U$ has only one maximum point which is a nondegenerate critical point of $U$ at 0 , we get that for any $\rho>0$, when $n$ is sufficiently large $u_{n}$ has only one maximum in $B_{\rho}(0)$ and therefore $w_{n}$ has only one maximum in $B_{\lambda_{n}, \rho}\left(y_{n}\right)$. This is by now a standard argument following [24]. Therefore there is a sequence $\rho_{n} \leqslant r_{n}$ satisfying $\rho_{n} \rightarrow \infty$ as $n \rightarrow \infty$ such that $y_{n}$ is the only local maximum of $w_{n}$ in $B_{\lambda_{n}, \rho_{n}}\left(y_{n}\right)$. Up to a subsequence, 
we get by a direct calculation and by (30)

$$
\begin{aligned}
S_{p}\left(\mathbf{R}^{N}\right)^{p /(p-2)} & =\lim _{n \rightarrow \infty} \int_{\mathbf{R}^{N}}|\nabla u|^{2}+u^{2} \leqslant \lim _{n \rightarrow \infty} \int_{B_{\rho_{n}}(0)}\left|\nabla u_{n}\right|^{2}+u_{n}^{2} \\
& =\lim _{n \rightarrow \infty} \int_{B_{\lambda_{n}, \rho_{n}}\left(y_{n}\right)}\left|\nabla w_{n}\right|^{2}+w_{n}^{2} \mathrm{~d} \mu=\lim _{n \rightarrow \infty} \int_{B_{\lambda_{n}, \rho_{n}}\left(y_{n}\right)} w_{n}^{p} \mathrm{~d} \mu .
\end{aligned}
$$

By assumption, let $\left\{y_{n, 1}, y_{n, 2}, \ldots, y_{n, k+1}\right\}$ maximum points of $w_{n}$. Performing the argument above at each of these points, we may assume for the same $\rho_{n}$

$$
B_{\lambda_{n}, \rho_{n}}\left(y_{n, i}\right) \cap B_{\lambda_{n}, \rho_{n}}\left(y_{n, j}\right)=\emptyset, \quad \text { for } i \neq j .
$$

From (29), (32), Propositions 4.3 and 4.4 we get

$$
k S_{p}\left(\mathbf{R}^{N}\right)^{p /(p-2)}=\lim _{n \rightarrow \infty} \int_{\mathcal{C}_{\lambda_{n}}}\left|\nabla w_{n}\right|^{2}+w_{n}^{2} \mathrm{~d} \mu \geqslant(k+1) S_{p}\left(\mathbf{R}^{N}\right)^{p /(p-2)} .
$$

This provides the desired contradiction.

Now, we argue that the number of local maxima is exactly $k$, and they form a $G$-orbit in $\Omega$. Indeed, from (32), (28) and (33), we obtain

$$
\lim _{n \rightarrow \infty} \int_{B_{\lambda_{n}, \rho_{n}}\left(y_{n}\right)} v_{n}^{p} \mathrm{~d} \mu \geqslant \frac{1}{k}
$$

Since

$$
\int_{\Lambda_{n}} v_{n}^{p} \mathrm{~d} \mu \rightarrow 1
$$

it follows that for $n$ sufficiently large, $y_{n} \in \Pi_{n}$. This implies there are at least $k$ local maxima. By (32), this in turn also implies $y_{n} \in \Omega_{n}$, for otherwise we would have

$$
\liminf _{n \rightarrow \infty} \int_{\Lambda_{n}} v_{n}^{p} \mathrm{~d} \mu>1
$$

The fact that local maxima form an orbit is a consequence of the fact that $w_{\lambda}$ has exactly $k$ local maximum points, and at least one of them is in $\Omega_{\lambda}$.

Part (iii) follows by the arguments in (ii), and the fact that $v_{\lambda}$ is a minimizer in $\mathcal{K}_{G, \lambda}^{\sigma}$, which implies for $r=r(\lambda) \rightarrow \infty$ with $r(\lambda) / \lambda \rightarrow 0$ as $\lambda \rightarrow \infty$

$$
\int_{\mathcal{C}_{\lambda} \backslash \bigcup_{i=1}^{k} B_{\lambda, r(\lambda)}\left(y_{\lambda, i}\right)}\left|\nabla v_{\lambda}\right|^{2}+v_{\lambda}^{2} \mathrm{~d} \mu \rightarrow 0, \quad \text { as } \lambda \rightarrow \infty .
$$

Taking $r(\lambda)=\sqrt{\lambda}$, we get the result. 
Part (iv) is direct consequence of Proposition 4.4.

Finally, (v) follows from (ii) since $\Sigma_{w}>\tilde{G}$ implies $w$ has more than $k$ local maxima.

Proof of Theorem 1.4. - Part (ii) was proved in [10]. The modified inversion symmetry in $\mathbf{R}^{N}$, corresponds to the even symmetry in $x_{N}$ on $\mathcal{C}$. The rest of the properties in Theorem 1.4 follow from Theorem 4.1 and transformations (5) and (7).

Proof of Theorem 1.1. - For $N \geqslant 2$, we write $\mathbf{R}^{N}=\mathbf{R}^{2} \times \mathbf{R}^{N-2}$ and for $k \geqslant 3$ consider $G_{k}=\mathbf{Z}_{k} \times \mathbf{O}(N-2)$ or $\mathbf{D}_{k} \times \mathbf{O}(N-2)$. For these two group actions, the minimal orbit set has this $k$ as the number of points in each orbit.

\section{Closing remarks}

Remark 5.1. - We mention that although for $N=2$ and $N \geqslant 4$ one can obtain the multiplicity result in Theorem 1.1 just by finding the minimum of $I_{\lambda}$ in the space $\mathcal{H}_{G_{k}, \lambda}$, our local minimization procedure in the space of symmetric functions is essential in order to obtain Theorems 1.1 and 1.4 in the case $N=3$. For $N=3$, a global minimization will only yield solutions with 1-bump (ground state), 2-bump (given by $\mathbf{Z}_{2} \times \mathbf{O}(2)$ symmetry), 4-, 6-, 12-bump (Example 2.3), and one radial solution. This phenomenon is similar to that for an elliptic problem on expanding annular domains studied in $[4,8,12,19,23]$. For the examples of higher dimensional tetrahedron or cube groups given in Example 2.6 with $N \geqslant 4$, a local minimization method is also needed if $l=N-1$. In these cases, the global minimization only produces 2-bump solutions.

Remark 5.2. - For the exact symmetry of solutions, we need the maximality condition (Definition 1.3), which seems necessary in some cases. For instance, the group $\mathbf{Z}_{k}$ given in Example 2.1 is not maximal with respect to the locally minimal orbit set there, and we believe in this case the solution one gets from Theorem 1.4 has exact symmetry $\mathbf{D}_{k}$ instead of $\mathbf{Z}_{k}$. One may compare with another problem of similar spirit studied in [17].

Remark 5.3. - In a forthcoming paper we shall present a multiplicity result of a somewhat different nature, where the imposed group of symmetry is continuous subgroup of $\mathbf{O}(N)$. These symmetries also make easier regaining of compactness and allow a larger parameter range.

\section{REFERENCES}

[1] Aubin T., Problèmes isopérimétriques de Sobolev, J. Differential Geom. 11 (1976) 573-598.

[2] Berestycki H., Esteban M., Existence and bifurcation of solutions for an elliptic degenerate problem, J. Differential Equations 134 (1997) 1-25.

[3] Brezis H., Lieb E.H., A relation between pointwise convergence of functions and convergence of functionals, Proc. Amer. Math. Soc. 88 (1983) 486-490.

[4] Byeon J., Existence of many nonequivalent nonradial positive solutions of semilinear elliptic equations on three-dimensional annuli, J. Differential Equations 136 (1997) 136-165.

[5] Caffarelli L.A., Gidas B., Spruck J., Asymptotic symmetry and local behavior of semilinear elliptic equations with critical Sobolev growth, Comm. Pure Appl. Math. 42 (1989) 271297. 
[6] Caffarelli L.A., Kohn R., Nirenberg L., First order interpolation inequalities with weights, Compositio Mathematica 53 (1984) 259-275.

[7] Caldiroli P., Musina R., On the existence of extremal functions for a weighted Sobolev embedding with critical exponent, Cal. Var. and PDEs 8 (1999) 365-387.

[8] Catrina F., Wang Z.-Q., Nonlinear elliptic equations on expanding symmetric domains, J. Differential Equations 156 (1999) 153-181.

[9] Catrina F., Wang Z.-Q., On the Caffarelli-Kohn-Nirenberg inequalities, C. R. Acad. Sci. Paris Sér. I Math. 300 (2000) 437-442.

[10] Catrina F., Wang Z.-Q., On the Caffarelli-Kohn-Nirenberg inequalities: sharp constants, existence (and nonexistence) and symmetry of extremal functions, Comm. Pure Appl. Math., in press.

[11] Chou K.S., Chu C.W., On the best constant for a weighted Sobolev-Hardy inequality, J. London Math. Soc. 2 (1993) 137-151.

[12] Coffman C.V., A nonlinear boundary value problem with many positive solutions, J. Differential Equations 54 (1984) 429-437.

[13] Dautray R., Lions J.-L., Mathematical Analysis and Numerical Methods for Science and Technology, Vol. 1, Springer-Verlag, Berlin, 1985.

[14] Gidas B., Ni W.-M., Nirenberg L., Symmetry of positive solutions of nonlinear elliptic equations in $R^{n}$, Adv. Math., Suppl. Studies 7A (1981) 369-402.

[15] Gilbarg D., Trudinger N.S., Elliptic Partial Differential Equations of Second Order, Springer, New York, 1998.

[16] Horiuchi T., Best constant in weighted Sobolev inequality with weights being powers of distance from the origin, J. Inequal. Appl. 1 (1997) 275-292.

[17] Kawohl B., Rearrangements and Convexity of Level Sets in PDE, Lecture Notes in Math., Vol. 1150, Springer, 1985.

[18] Kwong M.K., Uniqueness of positive solutions of $\Delta u-u+u^{p}=0$ in $R^{n}$, Arch. Rat. Mech. Anal. 105 (1989) 243-266.

[19] Li Y.Y., Existence of many positive solutions of semilinear elliptic equations on annulus, J. Differential Equations 83 (1990) 348-367.

[20] Lieb E.H., Sharp constants in the Hardy-Littlewood-Sobolev and related inequalities, Ann. Math. 118 (1983) 349-374.

[21] Lions P.-L., Concentration compactness principle in the calculus of variations. The locally compact case. Part 1, Ann. Inst. H. Poincaré Anal. Nonlinéaire 1 (1984) 109-145.

[22] Lions P.-L., Concentration compactness principle in the calculus of variations. The limit case. Part 1, Rev. Mat. Ibero. 1.1 (1985) 145-201.

[23] Mizoguchi N., Suzuki T., Semilinear elliptic equations on annuli in three and higher dimensions, Houston J. Math. 1 (1996) 199-215.

[24] Ni W.-M., Takagi I., On the shape of least-energy solutions to a semilinear Neumann problem, Comm. Pure Appl. Math. 45 (1991) 819-851.

[25] Palais R., The principle of symmetric criticality, Comm. Math. Phys. 69 (1979) 19-30.

[26] Talenti G., Best constant in Sobolev inequality, Ann. Mat. Pure Appl. 110 (1976) 353-372.

[27] Wang Z.-Q., Existence and symmetry of multi-bump solutions for nonlinear Schrödinger equations, J. Differential Equations 159 (1999) 102-137.

[28] Wang Z.-Q., Willem M., Singular minimization problems, J. Differential Equations 161 (2000) 307-320.

[29] Willem M., Minimax Theorems, Birkhäuser, Boston, 1996.

[30] Willem M., A decomposition lemma and critical minimization problems, preprint. 